\title{
The Magnitude of the Impact of HIV on the Human Beings in the World's Richest Country, America: The Misery of African Americans
}

\author{
Muse Abdi
}

Saint Mary's University of Minnesota 


\begin{abstract}
Disproportionate rates of HIV infection among African Americans is an increasing concern in the United States. The purpose of this study is to investigate the effect of HIV prevention programs on African Americans and social determinants fueling HIV-related risk behaviors. Using literature, this study analyzed the incidences of HIV infection among African Americans in the United States and the effectiveness of the prevention programs. African Americans struggle with mass incarceration, drugs, stigma, criminalization, and lack of economic opportunities, which contribute to the HIV-related risk behaviors. The existing traditional prevention programs in place are not working for African Americans. Tailored and culturally relevant programs should be designed and implemented. Further studies are needed to establish the causal relationships and develop preventive measures.
\end{abstract}




\section{The Magnitude of the Impact of HIV on the Human Beings in the World's Richest Country, America: The Misery of African Americans}

HIV is an infectious disease that can infect any human being via bloodily fluids, including semen, vaginal fluid, breastmilk, and blood. It has impacted countries worldwide, and 38 million people live with HIV globally (WHO, 2019). HIV disproportionately impacts underdeveloped countries compared to the rest of the world, especially Sub-Saharan African Countries. There are no functioning public health departments in these countries. They cannot get HIV prevention resources. Therefore, many people are living with HIV/AIDS, and these HIV patients do not get enough medications and nutritional support. More than 14,000 people contract the virus every day, while more than 11, 000 people die due to the AIDS complication every day (Mbirimtengerenji, 2007). Nearly 1.2 million Americans live with HIV, and 1 in 7 Americans who may have the virus do not know it (CDC, 2018). HIV is more endemic in poor, sexually active, and minority groups (Ruiz-Perez et al., 2017). While HIV is a global public health threat that impacts poor and sexually active groups in underdeveloped countries, here in America, the CDC and its partners deal with disproportionately impacted minority groups.

Although the CDC is the major organization involved in HIV prevention and treatment, many other organizations are involved in the fighting against HIV/AID: National Association of State and Territorial AIDS Directors (NASTAD), Urban Coalition for HIV/AIDS Prevention Services (UCHAPS), National Minority AIDS Council (NMAC), National Association of County and City Health Officials (NACCHO), The AIDS Institute, and local, state, and territorial health departments. Also, the American Red Cross and other nonprofit organizations participate this public health campaign. The mission of these agencies in HIV prevention is to educate, screen, 
and treat Americans with CDC as their primary reference point. However, the CDC gives general guidance towards HIV control.

HIV is a global public health threat that has no vaccine or cures. It is a communicable disease. Therefore, learning and addressing this topic is very important because the only pathway to control this infectious disease is to increase public awareness, reduce health disparities, and educate the public. Many people contract HIV because of a lack of HIV prevention resources or lack of knowledge. With the right education and enough resources, HIV transmission can be reduced to a level where it becomes eradicated.

HIV can impact any person regardless of age, gender, location, and socioeconomic. However, it is more common in the low-income minority and sexually active groups in the south (CDC, 2019). For example, in America, HIV is more prevalent among the poor and minority communities, such as African Americans, gay men, and Latinos (Holtgrave, 2015). These groups may not have housing, quality healthcare, and access to HIV prevention resources. They struggle with poverty, drugs, stigma, discrimination, fear, and homophobia, which can contribute to the rate of infection (CDC, 2020). Similarly, sexually active groups engage in more sexual activities compared to older or less sexually active groups. These groups' risk can be higher than the rest of the people unless they get appropriate resources.

African Americans contract HIV infection disproportionately more than other Americans. They suffer higher morbidity and mortality rates than other ethnic and racial groups in the United States. The most substantial portion of HIV diagnoses are African Americans because they may not receive quality healthcare and may lack confidence in the healthcare system. Moreover, governmental mass incarceration and criminalization of African Americans exacerbates the problem. To address the problem, prevention programs have been developed to reduce HIV rates. 
The primary purpose of this research was to answer the question, "How do prevention programs affect HIV infection in African Americans?" The following literature review describes the magnitude of HIV's impact on African Americans and the weaknesses of the prevention programs in place. After review, culturally relevant policies and suitable prevention interventions are necessary to combat this issue.

\section{Defining the Problem}

The percentage of individuals infected with HIV is higher among the African American population than it is among other racial groups in the United States. As recently as 2020, Essuon and his co-authors examined the impact of HIV on African Americans and found this population to have the highest percentage of new diagnoses of HIV. The authors presented that $44 \%$ of people living with HIV/AIDs are African Americans even though they only make up $13 \%$ of the total population of the United States. Similarly, an article by Gardner et al. (2019) discovered that HIV infection is disproportionately impacting African Americans and presented that $44 \%$ of HIVinfected cases are related to African Americans while they only represent $12 \%$ of the U.S. population. This study also found out that African Americans' HIV infection rate is six times higher than that of their white counterparts.

The death rate of HIV-infected African Americans continues to increase even though the rate of HIV infection in the United States is in decline. Robillard et al. (2017) noted that African Americans are disproportionately affected by HIV infection. The study stated that African Americans constitute the largest group infected by HIV with $44 \%$ of all HIV-infected cases being related to African Americans. The study also reported that the highest incidences of HIV infection are in the African Americans of the South. The article presented that $46.9 \%$ of all HIV-related deaths in the United States are associated with African Americans. Similarly, Danielson et al. 
(2016), who studied disproportionate risk of HIV infection on African Americans, found that the HIV diagnosis rate among African Americans is eight times that of whites. Another article by Woods-Jaeger et al. (2015) examined Wake County, the home of the state's capital, Raleigh, North Carolina and found 58\% of the HIV-infected individuals are African Americans while they constitute only $20.5 \%$ of the total population.

\section{The Root Cause of the Problem}

The root cause of HIV infection in the African American population is not only a lack of health services, stigma, and criminalization but also the lack of economic opportunities. According to Sangaranmoorthy et al. (2019), African Americans are disproportionately impacted by poverty, the absence of health insurance, lack of economic opportunities, and less viral suppression treatment adherence. The authors stated that African Americans struggle with stigma and discrimination, which leads to less retention in antiviral treatment programs and a lack of confidence in the overall healthcare system. The authors suggested that although the advancement of HIV prevention and treatment programs has decreased HIV infection in the United States, African Americans still suffer from high morbidity and mortality rates due to HIV infection. The article noted that structural and social drivers, such as public health policies, poverty, cultural norms, stigma, discrimination, and drug use are the causes behind this disproportionality. Similarly, Geter et al. (2018) found that African Americans live in poor conditions compared to other racial-ethnic groups in the United States, and the social and structural determinants are the barriers causing this unique problem for African Americans. The authors also stated that lack of family and social support, poor-quality HIV services, and African Americans' HIV related stigma from health providers contribute to HIV infection.

\section{Unjust Policing, Incarceration, and Criminalization Leading to High HIV Infections}


The role of public health is to reduce HIV infection in African Americans, yet for African Americans, government policies have often exacerbated the epidemic. For example, Kerr et al. (2016) studied the Drug War HIV/AIDS Inequities Model, an argument that disparities in HIV infection rates are caused by incarceration and state violence, and showed the ongoing criminal practices that increase African Americans' vulnerability to HIV infection. The authors remarked that the authorities indulge in malpractices that pose a risk of HIV transmission and spread stigma, such as mass incarceration and concentrated correctional facilities without safety resources. Similarly, Rowell-Cunsolo et al. (2016) suggested that African Americans are usually concentrated in correctional facilities, which increases the chances of risky behaviors such as unsafe sexual contact and illicit drug use. The authors highlighted that prisoners who are caught participating in illicit drug use are punished by depriving them of safe resources. Therefore, infected patients keep spreading the infection to other prisoners. Additionally, Kerr et al. (2016) stated that unjust policing, mass incarceration, criminalization, and HIV health disparities have inflamed the spread of HIV infection in African Americans.

\section{Policies and Programs Targeting HIV and the Disproportional Burden of Disease for}

\section{African Americans}

Most HIV prevention programs show little or no success in reducing HIV infection in African Americans. For instance, the article by Essuon et al. (2020) showed that Ending the HIV Epidemic (EHE), developed by the Trump Administration, was started to reduce HIV infection by 90\% between 2020 and 2030. According to the study, phase one of EHE targeted 50 jurisdictions with more than $50 \%$ of HIV infections and noted that seven states were disproportionately impacted by HIV infection. Essuon and his co-author presented that $79 \%$ were African Americans in these high burden states. However, Woods-Jaeger et al. (2015), who studied HIV prevention 
programs, stated that although CDC-controlled prevention programs were put in place to prevent HIV infection, those were not successful due to the lack of confidence of the African Americans in the healthcare system. Similarly, Danielson et al. (2016) showed that 74 HIV/STI risk reduction and prevention interventions were initiated to reduce the HIV infection, one-third of which were intended to help with controlling HIV infection in African Americans and other minority groups. However, according to the study, these prevention programs required greater resources, such as more staff and funding, and, therefore, these programs became inaccessible to African Americans.

\section{The Need for Designing and Implementing Culturally Relevant HIV Policies}

Preventing HIV infection in the African American population requires a different approach and greater action by the government, public health, and healthcare agencies. Okora et al. (2016) suggested that building a better relationship between healthcare providers and patients is an effective way to increase African American's retention and confidence in the healthcare system. The authors stated that if providers understand African Americans' special needs, they can provide the best care for the African American community. Likewise, Sangaranmoorthy et al. (2019) suggested creating tailored policies and culturally relevant preventive programs for African Americans to increase the retention of patients in the viral suppression treatment and increase the African Americans' confidence in the healthcare system. Moreover, Geter et al. (2018) highlighted the necessity for positive and better case management, support services, and mental health awareness to reduce HIV infection in African Americans. Additionally, the study suggested health provider training with respect to the special health needs of African Americans. For example, case management that integrates poverty alleviation or mental health approaches addressing the unique systemic violence of prison and policing experienced by African Americans. 


\section{Searching for Solutions: Alternative Pathways for Combatting the Impact of HIV Infection on African Americans}

Traditional prevention interventions are not working for African Americans, but other alternatives may work much better in reducing HIV infection. Woods-Jaeger et al. (2015) studied the effectiveness of a faith-based prevention program and found that faith-based HIV prevention programs increase the confidence of the African Americans in the health system and contribute to reducing the HIV infection in the community. Similarly, Gardner et al. (2019) used a series of quantitative surveys to assess the readiness of African Americans toward HIV prevention programs in barbershop sites in the south, and the authors noted that HIV prevention programs delivered at barbershops are effective in reducing the risks faced by African Americans. Gardner and his colleagues remarked that barbershop sites are common venues where African Americans convene. In the same way, Robillard et al. (2017) studied culture-centric prevention programs that used storytelling sessions for African Americans. The authors used HIV positive patients to warn the other community members regarding the risk posed by the infection. Moreover, the authors directed the African American community to the preventive resources available to them. According to the study, these actions drew the African American community's attention to the risks posed by HIV infection, and the authors emphasized the importance of storytelling sessions, suggesting that these sessions could reduce the behaviors causing the risk of HIV infection, such as unprotected sex and drug-related HIV spread.

Technology-based HIV prevention programs can be implemented too. These programs have shown excellent success in reducing HIV infection among African Americans. For example, Murry et al. (2019) examined a technology-delivered HIV prevention program targeting African American families. The authors said that prevention programs utilized family discussion, sharing 
norms, and parental knowledge regarding the risk of HIV infection, and the results of the study showed promising and decreasing risk behaviors among African Americans. Likewise, Danielson et al. (2016) stated that internet-delivered HIV prevention programs showed decreasing HIV risk behaviors among African Americans compared to the effectiveness of existing traditional prevention programs. Additionally, Danielson and his co-author said that HIV prevention delivered through the internet reaches more folks compared to the existing methods, and they observed increasing awareness among African Americans from the use of the same.

\section{Summary and Recommendations}

HIV infection disproportionally impacts African Americans. Although African Americans account for only $13.4 \%$ of the U.S. population, they make up $44 \%$ of the HIV-infected population. Poverty, drugs, criminalization, incarceration, and health disparities are major contributors to HIV infections in the African American community. Traditional HIV prevention programs have shown no success in reducing infection rates. However, experts have suggested that culturally appropriate programs and technology-based preventions show promising results.

Although the literature reviewed in this paper defines the overall problem and offers some solutions, further research is required to more clearly classify the problems caused by HIV infections and to develop sustainable solutions for African Americans. Specifically, research needs to examine the impact of prevention programs that integrate solutions that alleviate poverty and stigma as a way to prevent HIV infection. Additionally, research should focus on ways to eliminate healthcare and provider bias that exacerbates HIV infection rates. Research has shown that prevention must be culturally appropriate. Given the under-representation of African Americans in medicine, research needs to evaluate ways to eliminate bias and assumptions that contribute to prevention efforts that are not culturally appropriate. Addressing the disparities in HIV infection 
rates requires a focus on three levels: patient, provider, and infrastructure. Future research and interventions must address all three levels in order to curb the disproportional burden of disease among African Americans in the United States. 


\section{References}

Bailey, M. E. (1991). Community-based organizations and CDC as partners in HIV education and prevention. Public Health Reports (Washington, D.C.: 1974), 106(6), 702-708.

CDC. (2018). Estimated HIV incidence and prevalence in the United States. Center for Disease Control. https://www.cdc.gov/hiv/pdf/library/reports/surveillance/cdc-hiv-surveillancesupplemental-report-vol-25-1.pdf

CDC. (2019.). Advancing the national HIV/AIDS strategy. Center for Disease Control. https://www.cdc.gov/hiv/programresources/healthdepartments/index.html\#

Danielson, C. K., McCauley, J. L., Gros, K. S., Jones, A. M., Barr, S. C., Borkman, A. L.,Bryant, B. G., \& Ruggiero, K. J. (2016). SiHLEWeb.com: Development and usability testing of an evidence-based HIV prevention website for female African-American adolescents. Health Informatics Journal, 22(2), 194208. https://doi.org/10.1177/1460458214544048

Essuon, A. D., Zhao, H., Wang, G., Collins, N., Karch, D., \& Rao, S. (2020). HIV testing outcomes among Blacks or African Americans - 50 Local U.S. jurisdictions accounting for the majority of new HIV diagnoses and seven states with disproportionate occurrences of HIV in rural areas, 2017. MMWR. Morbidity and Mortality Weekly Report, 69(4), 97-102. https://doi-org.xxproxy.smumn.edu/10.15585/mmwr.mm6904a2

Gardner, A. J., Paschal, A. M., Leeper, J., Usdan, S., Gordon, B., \& Tucker, M. T. (2019). Assessing readiness for barbershop-based HIV prevention programs in the South. American Journal of Health Studies, 34(4), 214-221. 
Geter, A., Sutton, M. Y., \& Hubbard McCree, D. (2018).Social and structural determinants of HIV treatment and care among black women living with HIV infection: a systematic review: 2005-2016. AIDS Care, 30(4), 409-416. https://doiorg.xxproxy.smumn.edu/10.1080/09540121.2018.1426827

Holtgrave, D. R. (2015). Achieving and advancing the goals of the National HIV/AIDS Strategy for the United States. AIDS and Behavior, 19(2), 211-213. https://doiorg.xxproxy.smumn.edu/10.1007/s10461-014-0903-z

Kerr, J., \& Jackson, T. (2016). Stigma, sexual risks, and the war on drugs: Examining drug policy and HIV/AIDS inequities among African Americans using the Drug War HIV/AIDS Inequities Model. International Journal of Drug Policy, 37, 31-41. https://doi-org.xxproxy.smumn.edu/10.1016/j.drugpo.2016.07.007

Mbirimtengerenji N. D. (2007). Is HIV/AIDS epidemic outcome of poverty in sub-Saharan Africa? Croatian medical journal, 48(5), 605-617.

Murry, V. M., Kettrey, H. H., Berkel, C., \& Inniss-Thompson, M. N. (2019). The pathways for African American success: Does delivery platform matter in the prevention of HIV risk vulnerability among youth? Journal of Adolescent Health, 65(2), 255261. https://doi.org/10.1016/j.jadohealth.2019.02.013

Okoro, O., \& Odedina, F. T. (2016). Improving medication adherence in African-American women living with HIV/AIDS: Leveraging the provider role and peer involvement. AIDS Care, 28(2), 179-185. https://doiorg.xxproxy.smumn.edu/10.1080/09540121.2015.1071771 
Robillard, A., Padi, A., Lewis, K., Julious, C., \& Troutman, J. (2017). Advice for prevention from HIV-positive African-American women: "My story is not just a story." Culture, Health Sexuality, 19(5), 630-642. https://doi.org/10.1080/13691058.2016.1243732

Rowell-Cunsolo, T. L., El-Bassel, N., \& Hart, C. L. (2016). Black Americans and incarceration: A neglected public health opportunity for HIV risk reduction. Journal of Health Care for the Poor and Underserved, 27(1), 114-130. https://doiorg.xxproxy.smumn.edu/10.1353/hpu.2016.0011

Ruiz-Perez, I., Murphy, M., Pastor-Moren, G., Rojas-García, A., \& Rodríguez-Barranco, M. (2017). The effectiveness of HIV prevention interventions in socioeconomically disadvantaged ethnic minority women: A systematic review and meta-analysis. American Journal of Public Health, 107(12), e13-e21. https://doiorg.xxproxy.smumn.edu/10.2105/AJPH.2017.304067

Sangaramoorthy, T., Jamison, A., \& Dyer, T. (2019). Older African Americans and the HIV care continuum: A systematic review of the literature, 2003-2018. AIDS and Behavior, 23(4), 973-983. https://doi org.xxproxy.smumn.edu/10.1007/s10461-018-2354-4

Woods-Jaeger, B., Carlson, M., Taggart, T., Riggins, L., Lightfoot, A., \& Jackson, M. (2015). Engaging African American faith-based organizations in adolescent HIV prevention. Journal of Religion \& Health, 54(4), 1358-1374. https://doiorg.xxproxy.smumn.edu/10.1007/s10943-014-9932-1 